\title{
P01-3-31 Poster session
}

\section{The role of rho-kinase in phenylephrine and 5-HT-induced smooth muscle contractions in the porcine ureter}

\author{
Iris Lim, Russ Chess-Williams, Donna Sellers \\ Centre for Urology Researh, Bond University, Australia
}

Background. Urolithiasis is frequently accompanied by colic, which is understood to be caused by contractions of the ureteral smooth muscle. Since calcium sensitisation via the rho-kinase pathway plays a significant role in smooth muscle contraction, our aim was to investigate the role of Rho-kinase in phenylephrine and 5-HT-induced contractile activity in isolated ureters from young and older animals.

Methods. Contractile responses of isolated smooth muscle strips to EC50 (low) and maximal (high) doses of phenylephrine and 5-HT were examined in distal ureter from young (6 months) and old (3 years) pigs s in the absence and presence of Rho-kinase inhibitors, fasudil (30\&muM) or Y-27632 (10\&muM). Tissues developed phasic contractions and were expressed as area under the ,normalized to tissue weight. Additionally, isolated ureteral smooth muscle strips were homogenized, and rho-kinase activity assay kit was utilised to measure rho-kinase activity at basal, phenylephrineand 5-HT-induced states.

Results. Fasudil significantly attenuated ureteral contractions to low and high doses of phenylephrine by $81.9 \pm 3.0 \%$ and $89.8 \pm 9.2 \%$ vs control response in tissues from young animals. In response to 5 -HT, fasudil reduced contractions by 83.7 $\pm 6.2 \%$ and $77.7 \pm 3.2$ vs control response at low and high doses respectively. In tissues from older animals, similar effects were observed, where contractions to low and high doses of phenylephrine were reduced by $84.0 \pm 3.9 \%$ and $78.7 \pm 3.0 \%$ vs control response. Contractions to 5 -HT were also reduced by $80.1 \pm 6.9 \%$ and $79.3 \pm 3.2 \%$ vs control response at low and high doses respectively. Y-27632 produced similar effects with both agonists in tissues from young and old animals. Active rho-kinase activity was similar in both age groups at basal and agonists-induced states.

Conclusions. Our results show that rho-kinase mediates contractile responses to phenylephrine and 5-HT in isolated ureteral tissues of the pig and may be a potential target for development of treatments aimed at relaxing ureteral smooth muscle. 